\title{
Experimental fitting to the bipolaronic model of the normal-state resistance of $\mathrm{Bi}_{2} \mathrm{Sr}_{2} \mathrm{CaCu}_{2} \mathrm{O}_{8}$ single crystals*
}

\author{
Weimin Chen, J. P. Franck, and J. Jung \\ Department of Physics, University of Alberta, Edmonton, Canada T6G 2J1
}

\begin{abstract}
Normal-state resistance data from $\mathrm{Bi}_{2} \mathrm{Sr}_{2} \mathrm{CaCu}_{2} \mathrm{O}_{8}$ single crystals were fitted to the (bi)polaronic conduction model, $R=R_{0}\left(T+\sigma_{b} T^{2}\right) /(1+b T)$, with satisfactory agreement over a wide temperature range. The fluctuating conduction region is found to be much narrower than that in the usual sense, as is the case for a charged Bose-gas. We estimate the effective (bi)polaron mass to be $\sim 4 m_{e}$.
\end{abstract}

We report on the normal-state resistance measurement of $\mathrm{Bi}_{2} \mathrm{Sr}_{2} \mathrm{CaCu}_{2} \mathrm{O}_{8}$ (Bi-2212) single crystals and its fitting to the bipolaron model [1.2]. We found that the data fitted to this theory over wide temperature ranges. In some samples, the fitting spans from about $T_{c}+40 \mathrm{~K}$ up to 300 $\mathrm{K}$. Moreover, since the fitting range extends down to low temperatures near $T_{c}$, the superconducting fluctuation region is much narrower than that in the usual sense. These results provide alternative interpretations of the peculiar behavior of the normal-state resistivity.

The samples studied in the present work were Bi-2212 single crystalline whiskers [3], with typical dimensions of 2 to $3 \mathrm{~mm} \times 10 \times 0.5 \mu \mathrm{m}$ along the $a, b$, and $c$ axes, respectively. Resistance was measured by the standard four-wire method in the $a b$-plane with a dc current of $0.5 \mu \mathrm{A}$.

Figure 1 shows a typical plot of resistance data. The dotted line is a linear- $T$ fit at high temperature. As usual, the resistance shows a downward deviation around $200 \mathrm{~K}$, which is generally associated with the pseudogap phenomenon (or superconducting fluctuation). In the bipolaronic scenario, on the other hand, the normal-state dynamics is dominated by small polarons and the superconducting state is a charged (2e) BoseEinstein condensate of bipolarons [1]. Quantita-

\footnotetext{
*We would like to thank A. S. Alexandrov for a critical reading of the manuscript. This research was supported by grants from the National Sciences and Engineering Research Council (NSERC) of Canada.
}

tively, this model gives for the resistance:

$$
R=R_{0} \frac{T+\sigma_{b} T^{2}}{1+b T}
$$

where $\sigma_{b}$ is the relative boson-boson scattering cross section, and $b$ is related to Hall coefficient,

$R_{H}=\frac{v_{0}}{2 e\left(n-n_{L}\right)(1+b T)}$,

with $\left(n-n_{L}\right)(1+b T)$ being the number of delocalized carriers in the unit-cell volume $v_{0}(5.40 \times$ $5.41 \times 30.9 \AA^{3}$ ), and $R_{0}$ is a fitting parameter.

By using the Hall coefficient data from Ref. [4]: $R_{H}\left(10^{-3} \mathrm{~cm}^{3} / \mathrm{C}\right)=2.5,2.3,2.1$, and 1.9 , for $T$ $=150,200,250$, and $300 \mathrm{~K}$, we obtained the values for $b=0.003 \mathrm{~K}^{-1}$ and $\left(n-n_{L}\right)=0.076$ from Eq. 2. This results in $\sigma_{b} \approx 0.0011$ to 0.0016 from initial fitting of six samples. Suppose $\sigma_{b}$ is less sensitive to oxygen doping than $b$, so we adopt constant $\sigma_{b}=0.0015$ in the fitting. The result is as shown by the solid line in Fig. 1. As can be seen, the data closely agrees with this model over a wide temperature range. Two features in Fig. 1 are noticeable: (1) While resistance deviation from the linear- $T$ fitting is evident, it is well accounted for in the polaron model; and (2) Superconducting fluctuation is seen only very close to $T_{c}$ in the polaron fitting, in contrast to the much higher onset temperature if the linear- $T$ criterion is used. The above analysis, together with recent experiments which showed very narrow coherent region above $T_{c}$ [5], does not seem to support superconducting fluctuation at high temperature $(\sim 200 \mathrm{~K})$. The coherence length obtained 


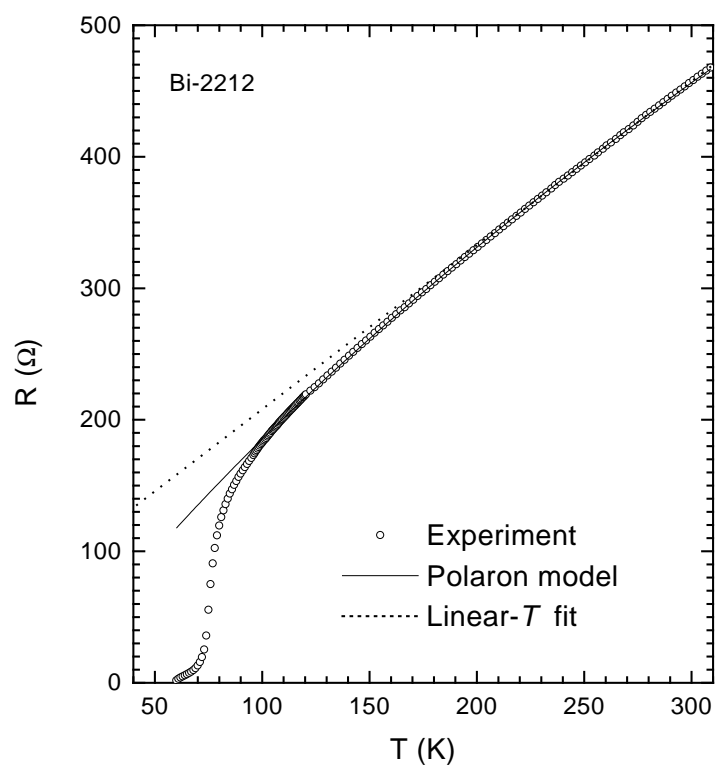

Figure 1. Linear- $T$ (dotted line) and bipolaronic (solid line) fittings to the normal-state resistance of a Bi-2212 sample. The latter covers a much wider region than the former.

by using the Aslamazov-Larkin model [6] agrees well with literature data. Therefore, we argue the superconducting fluctuation region in the present picture is much narrower than that in the usual sense. This point agrees with the scenario of a charged Bose-gas [7]. A similar conclusion was also established from transport measurement on YBCO [8].

The fitting results for three samples were shown in Fig. 2 (solid lines), all yielded mutual agreeable $b$ values. The paraconducting deviation happens at $\sim\left(T_{c}+50\right) \mathrm{K}$, which is otherwise much higher $(\sim 200 \mathrm{~K})$ as described. The numbers in the plot show the values of the fitting parameters $R_{0} / R(300 \mathrm{~K})$ and $b$, respectively.

To further check the validity of this approach, we calculate the effective mass of the in-plane boson mass [1], $m=\pi \hbar^{2} / w a^{2}$, where the lattice constant $a=5.4 \AA, w$ is related to $T_{c}$ through

$T_{c}=\frac{2 w\left(n-n_{L}\right)}{\log \gamma^{2}}$.



Figure 2. Resistance data for three Bi-2212 samples. The numbers are fitting parameters of $R_{0} / R(300 \mathrm{~K})$ and $b$ (refer to Eq. I1). Inset: details near $T_{c}$.

Take $T_{c}=75 \mathrm{~K}, \gamma=183$, and $\left(n-n_{L}\right)=0.076$, we have $m=4.27 \times$ free electron mass. Thus, the result may well correspond to small polarons and to inter-site bipolarons.

\section{REFERENCES}

1. A. S. Alexandrov, A. M. Bratkovsky, and N. F. Mott, Phys. Rev. Lett. 72 (1994) 1734; Supercond. Sci. Technol. 6 (1993) 755.

2. A. S. Alexandrov and P. E. Kornilovitch, Phys. Rev. Lett. 82 (1999) 807.

3. J. Jung et al., Jpn. J. Appl. Phys. 28 (1989) L1182.

4. Y. Iye et al., Physica C 159 (1989) 616.

5. J. Corson et al., Nature (London) 398 (1999) 221.

6. L. G. Aslamazov and A. I. Larkin, Phys. Lett. 26A (1968) 238.

7. A. S. Alexandrov (private communication).

8. V. F. Gantmakher et al., JETP 88 (1999) 148. 